\title{
Modeling of Path Nonparametric Truncated Spline Linear, Quadratic, and Cubic in Model on Time Paying Bank Credit
}

\author{
Erlinda Citra Lucki Efendi, Adji Achmad Rinaldo Fernandes*, Maria Bernadetha Theresia Mitakda
}

Department of Statistics, Faculty of Mathematics and Natural Sciences, Brawijaya University, Indonesia

Received August 26, 2021; Revised October 22, 2021; Accepted November 11, 2021

\begin{abstract}
Cite This Paper in the following Citation Styles
(a): [1] Erlinda Citra Lucki Efendi, Adji Achmad Rinaldo Fernandes, Maria Bernadetha Theresia Mitakda , "Modeling of Path Nonparametric Truncated Spline Linear, Quadratic, and Cubic in Model on Time Paying Bank Credit," Mathematics and Statistics, Vol. 9, No. 6, pp. 947 - 957, 2021. DOI: 10.13189/ms.2021.090611.
\end{abstract}

(b): Erlinda Citra Lucki Efendi, Adji Achmad Rinaldo Fernandes, Maria Bernadetha Theresia Mitakda (2021). Modeling of Path Nonparametric Truncated Spline Linear, Quadratic, and Cubic in Model on Time Paying Bank Credit. Mathematics and Statistics, 9(6), 947 - 957. DOI: 10.13189/ms.2021.090611.

Copyright $(\mathrm{C} 2021$ by authors, all rights reserved. Authors agree that this article remains permanently open access under the terms of the Creative Commons Attribution License 4.0 International License

\begin{abstract}
This study aims to estimate the nonparametric truncated spline path functions of linear, quadratic, and cubic orders at one and two knot points and determine the best model on the variables that affect the timely payment of House Ownership Credit (HOC). In addition, this study aims to test the hypothesis to determine the variables that have a significant effect on punctuality in paying House Ownership Credit (HOC). The data used in this study are primary data. The variables used are service quality and lifestyle as exogenous variables, willingness to pay as mediating variables and on time to pay as endogenous variables. Analysis of the data used in this study is a nonparametric path using $\mathrm{R}$ software. The results showed that the best model was obtained on a nonparametric truncated spline linear path model with 2 knot points. The model has the smallest GCV value of 25.9059 and $\mathrm{R}^{2}$ value of $96.96 \%$. In addition, the results of hypothesis testing on function estimation have a significant effect on the relationship between service quality and willingness to pay, the relationship between service quality and on time to pay, the relationship between lifestyle and willingness to pay, and the relationship between lifestyle and on time pay. The novelty of this research is to model and test the hypothesis of nonparametric regression development, namely nonparametric truncated spline paths of linear, quadratic and cubic orders.
\end{abstract}

Keywords Path Analysis, Path Nonparametric, Truncated Spline, House Ownership Credit

\section{Introduction}

In a study, researchers usually observe a relationship between variables. One method of statistical analysis that can be used is regression analysis, where regression analysis is a method used to observe the relationship between two or more variables and can be used to determine the pattern of the relationship from a model whose form is not yet known [1].

There are three regression analysis approaches that can be done, namely nonparametric, semiparametric, and parametric approaches [2]. In the nonparametric regression approach the form of the regression function is assumed to be unknown and the linearity assumption is not met, the parametric regression approach is assumed to be known and the linearity assumption is met [3], while the semiparametric regression approach is a combination of parametric and nonparametric regression that can be performed if some of the curves are assumed to be known and some are assumed to be unknown [4].

Many current studies use more than one dependent variable. If there are two dependent variables, regression cannot be performed. Path analysis or path analysis can overcome these weaknesses [5]. According to Sudaryono [6], path analysis is a method that observes the direct and indirect effects of the hypothesized variables. Path 
analysis is not a causal method (cause and effect), but a method that can be used for causal models formulated on basic knowledge and theories developed by researchers.

Often found in real life, the pattern of the relationship between the dependent and independent variables cannot be known in the shape of the curve [7]. Path analysis based on nonparametric regression is a regression approach or path analysis that is in accordance with the pattern of the relationship between the dependent variable and the independent variable whose curve shape is not known [8]. In addition, nonparametric regression approaches tend to be freer to search for regression patterns so that they are flexible and objective.

House Ownership Credit (HOC) is one of the credit service facilities provided by banks to customers to meet their needs in the form of housing. Banks in providing a credit facility to customers, one of the most considered is the quality service and lifestyle. The quality service to customers can be seen in terms of reliability, responsiveness, assurance, empathy, and tangibles. Meanwhile, the customer's lifestyle towards HOC decisions can be seen in terms of activities, interests, and opinions.

Referring to Husnawaty's research [9], states that service quality needs to be improved, especially with regard to reliability so that various complaints can be avoided by customers and strive to provide satisfaction to customers regarding the provision of HOC. With the provision of HOC by the bank, there will be possible risks faced, such as willingness to pay credit (willingness to pay) and on time in paying credit. Therefore, to reduce risk, the bank must be more selective and careful in providing loans to customers through an attitude assessment. The assessment is meant by linking the variables of Service Quality and lifestyle with Willingness to Pay (willingness to pay) and Punctuality to Pay. As expected in this study, in order to minimize the HOC risk faced by the bank, by improving the HOC service system, it creates a high willingness of customers to pay for HOC.

Based on the description above, this study will examine the estimation of nonparametric truncated spline path functions with linear, quadratic, and cubic orders at one and two knot points. This study aims to estimate and select the best model on the variables that affect the timely payment of HOC. In addition, this study aims to test the hypothesis to determine the variables that have a significant effect on punctuality in paying HOC. In estimating the truncated spline nonparametric path function, the Least Square (LS) approach is used to determine the variables that affect the punctuality of paying HOC.

\section{Materials and Methods}

\subsection{Research Data}

The data used in this study are primary data regarding service quality, lifestyle, willingness to pay, and on time to pay. Data obtained through questionnaires distributed to respondents who have been determined. Respondents in this study were customers of HOC Bank X in Sidoarjo City. The research instrument uses a Likert scale to measure the variables used. The sampling technique used is purposive sampling where the sampling is adjusted on the basis of certain characteristics or conditions that are the same as the population. The sample used in this study is 100 customers of HOC Bank X in 2021. The variables used are quality service $\left(\mathrm{X}_{1}\right)$, lifestyle $\left(\mathrm{X}_{2}\right)$, willingness to pay $\left(\mathrm{Y}_{1}\right)$, and on time to pay $\left(\mathrm{Y}_{2}\right)$.

\subsection{Research Model}

In this study, two exogenous variables were used, namely variables quality service $\left(\mathrm{X}_{1}\right)$, lifestyle $\left(\mathrm{X}_{2}\right)$, willingness to pay $\left(\mathrm{Y}_{1}\right)$, and on time to pay $\left(\mathrm{Y}_{2}\right)$. The path diagram for this study is presented in Figure 1.

\subsection{Research Procedure}

The steps taken in this study are as follows:

1. Make a path diagram based on the predetermined exogenous and endogenous variables.

2. Test the linearity assumption of the relationship between variables using Ramsey's Regression Specification Error Test (RESET) with a calculated F value. If the assumption of linearity is met, then parametric path analysis can be used. However, if the assumption of linearity is not met, it can be continued to the next stage.

3. Estimate the path function with truncated spline with linear, quadratic, and cubic orders with the number of knots, namely 1 point knot, and 2 point knot using the LS method.

4. Obtain the function estimation results of each degree of the polynomial, namely linear, quadratic, and cubic with the number of knots, namely 1 point knot, and 2 point knot.

5. Selection of the optimum model and the optimum knot point based on the smallest GCV coefficient.

6. Interpret the estimation results of the best truncated spline model nonparametric path function and conclude from the results of the analysis.

7. Perform hypothesis testing on the best model. 


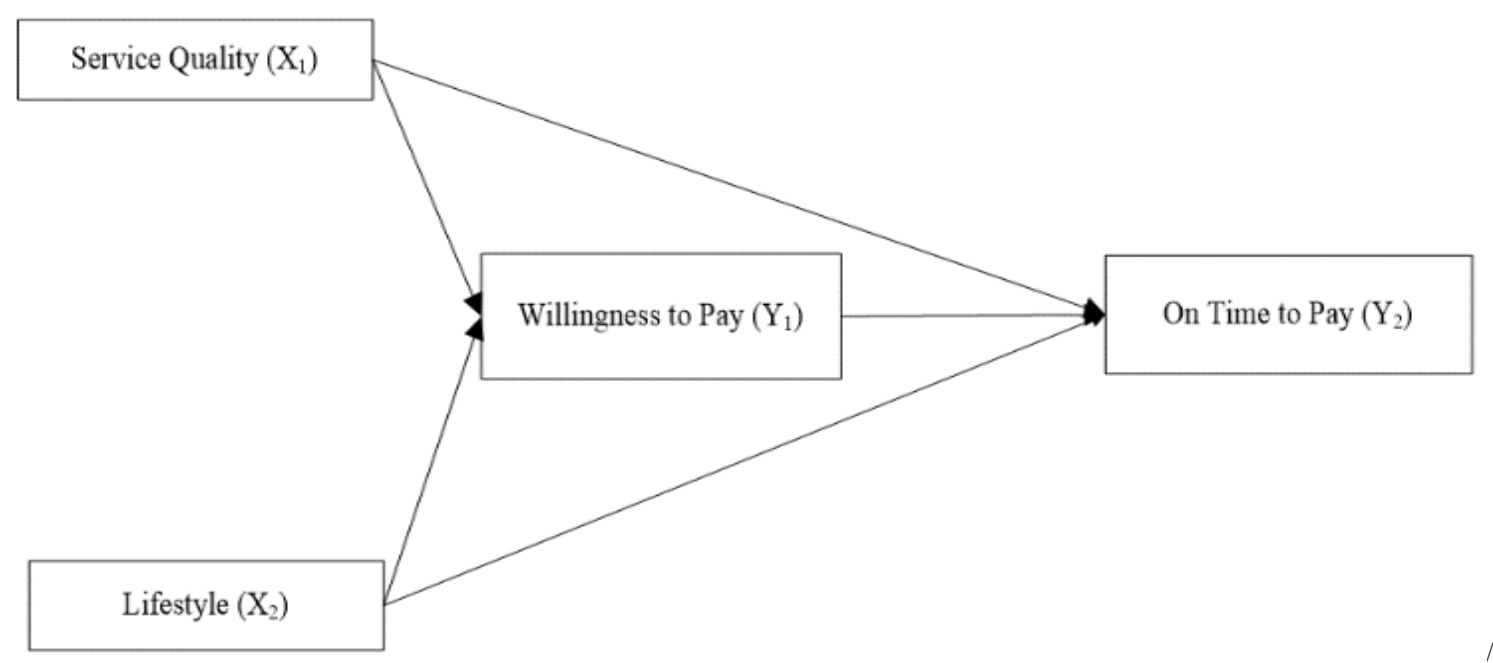

Figure 1. Research Path Diagram

\section{Result}

\subsection{Structural Model Development}

Lemma 3.1 Forms of the Linear Truncated Spline Nonparametric Path Model

If given paired data $\left(X_{1 i}, X_{2 i}, Y_{1 i}, Y_{2 i}\right)$ with $i=1,2, \ldots, n$ those following the nonparametric path analysis model, then the form of the linear nonparametric path analysis function is obtained as presented in equation (1) and the model in (2).

$$
\begin{gathered}
Y_{1 i}=f_{1}\left(X_{1 i}, X_{2 i}\right)+\varepsilon_{1 i} \\
Y_{2 i}=f_{2}\left(X_{1 i}, X_{2 i}, Y_{1 i}\right)+\varepsilon_{2 i} \\
Y_{1 \mathrm{i}}=\beta_{10}+\beta_{11} X_{1 i}+\delta_{11}\left(X_{1 i}-K_{11}\right)_{+}+\beta_{12} X_{2 i} \\
+\delta_{12}\left(X_{2 i}-K_{21}\right)_{+}+\varepsilon_{1 i} \\
Y_{2 \mathrm{i}}=\beta_{20}+\beta_{21} X_{1 i}+\delta_{21}\left(X_{1 i}-K_{11}\right)_{+}+\beta_{22} X_{2 i} \\
+\delta_{22}\left(X_{2 i}-K_{21}\right)_{+}+\beta_{23} Y_{1 i}+\delta_{23}\left(Y_{1 i}-K_{31}\right)_{+}+\varepsilon_{2 i}
\end{gathered}
$$

\section{Proof}

Before obtaining a model in the linear nonparametric path analysis, the first model is obtained from (a) multiple linear regression analysis; (b) simple linear path analysis; and (c) nonparametric regression analysis as follows:

First part:

It is known that the multiple linear regression model with equation (3) and the model in (4)

$$
\begin{gathered}
Y_{i}=f\left(X_{1}, X_{2}\right)+\varepsilon_{i} \\
Y_{i}=\beta_{0}+\beta_{1} X_{1}+\beta_{2} X_{2}+\varepsilon_{i}
\end{gathered}
$$

The second part:

It is known that the simple path analysis model is presented in equation (5) and the model in (6)

$$
\begin{gathered}
Y_{1 i}=f_{1}\left(X_{1 i}, X_{2 i}\right)+\varepsilon_{1 i} \\
Y_{2 i}=f_{2}\left(X_{1 i}, X_{2 i}, Y_{1 i}\right)+\varepsilon_{2 i} \\
Y_{1 \mathrm{i}}=\beta_{10}+\beta_{11} X_{1 i}+\beta_{12} X_{2 i}+\varepsilon_{1 i} \\
Y_{2 \mathrm{i}}=\beta_{20}+\beta_{21} X_{1 i}+\beta_{22} X_{2 i}+\beta_{23} Y_{1 i}+\varepsilon_{2 i}
\end{gathered}
$$

Part Three:

After knowing the equation and multiple linear regression models, a nonparametric regression model can be made as presented in Equations (7) and (8).

$$
\begin{gathered}
Y_{i}=f_{1}\left(X_{1 i}, X_{2 i}\right)+\varepsilon_{i} \\
Y_{\mathrm{i}}=\beta_{10}+\beta_{11} X_{1 i}+\delta_{11}\left(X_{1 i}-K_{11}\right)_{+}+\beta_{12} X_{2 i} \\
+\delta_{12}\left(X_{2 i}-K_{21}\right)_{+}+\varepsilon_{1 i}
\end{gathered}
$$

From the equations in the simple linear regression analysis model, simple path analysis, and nonparametric regression analysis that has been described, a function that is formed as in equations (1) and (2) can be obtained, so that the following matrix is obtained:

$$
\begin{aligned}
& \boldsymbol{Y}_{2 \mathrm{nx} 1}=\boldsymbol{X}_{2 n x 12} \boldsymbol{\beta}_{12 x 1}+\varepsilon_{2 n x 1} \\
& {\left[\begin{array}{c}
Y_{11} \\
Y_{12} \\
\vdots \\
Y_{1 n} \\
Y_{21} \\
Y_{22} \\
\vdots \\
Y_{2 n}
\end{array}\right]=\left[\begin{array}{cc}
\boldsymbol{X}_{\boldsymbol{X}} & \mathbf{0}_{n x 7} \\
\mathbf{0}_{n x 5} & \boldsymbol{X}_{\boldsymbol{X} Y}
\end{array}\right]\left[\begin{array}{c}
\beta_{01} \\
\beta_{11} \\
\delta_{11} \\
\beta_{12} \\
\delta_{12} \\
\beta_{20} \\
\beta_{21} \\
\delta_{21} \\
\beta_{22} \\
\delta_{22} \\
\beta_{23} \\
\delta_{23}
\end{array}\right] ;}
\end{aligned}
$$

Where, 


$$
\begin{gathered}
\boldsymbol{X}_{\boldsymbol{X}}=\left[\begin{array}{ccccc}
1 & X_{11} & \left(X_{11}-K_{11}\right)_{+} & X_{21} & \left(X_{21}-K_{21}\right)_{+} \\
1 & X_{12} & \left(X_{12}-K_{11}\right)_{+} & X_{22} & \left(X_{22}-K_{21}\right)_{+} \\
\vdots & \vdots & \vdots & \vdots & \vdots \\
1 & X_{1 n} & \left(X_{1 n}-K_{11}\right)_{+} & X_{2 n} & \left(X_{2 n}-K_{21}\right)_{+}
\end{array}\right] \\
\boldsymbol{X}_{\boldsymbol{X}}=\left[\begin{array}{ccccc}
1 & X_{11} & \left(X_{11}-K_{11}\right)_{+} & X_{21} & \left(X_{21}-K_{21}\right)_{+} \\
1 & X_{12} & \left(X_{12}-K_{11}\right)_{+} & X_{22} & \left(X_{22}-K_{21}\right)_{+} \\
\vdots & \vdots & \vdots & \vdots & \vdots \\
1 & X_{1 n} & \left(X_{1 n}-K_{11}\right)_{+} & X_{2 n} & \left(X_{2 n}-K_{21}\right)_{+} \\
& & & \\
& Y_{11} & \left(Y_{11}-K_{31}\right)_{+} \\
Y_{12} & \left(Y_{12}-K_{31}\right)_{+} \\
\vdots & \vdots \\
Y_{1 n} & \left(Y_{1 n}-K_{31}\right)_{+}
\end{array}\right]+\left[\begin{array}{c}
\varepsilon_{11} \\
\varepsilon_{11} \\
\vdots \\
\varepsilon_{1 n} \\
\varepsilon_{21} \\
\varepsilon_{22} \\
\vdots \\
\varepsilon_{2 n}
\end{array}\right]
\end{gathered}
$$

Where,

$f\left(X_{i j}\right)$ : the nonparametric regression function of the $i$ th observation of the $j$ th nonparametric exogenous variable

$X_{i j}$ : the exogenous variable $j$ th in the $i$ th observation

$\beta_{i j}$ : the parameter of the exogenous variable $j$ th in the $i$ th observation

$\delta_{i j}$ : parameter of the function truncated spline

Lemma 3.2 Form of Quadratic Truncated Spline Nonparametric Path Model

If given paired data $\left(X_{1 i}, X_{1 i}^{2}, X_{2 i}, X_{2 i}^{2}, Y_{1 i}, Y_{1 i}^{2}, Y_{2 i}, Y_{2 i}^{2}\right)$ with $i=1,2, \ldots, n$ those that follow the nonparametric path analysis model, then the quadratic nonparametric path analysis function is obtained as presented in equation (9) and the model in (10).

$$
\begin{gathered}
Y_{1 i}=f_{1}\left(X_{1 i}, X_{1 i}^{2}, X_{2 i}, X_{2 i}^{2}\right)+\varepsilon_{1 i} \\
Y_{2 i}=f_{2}\left(X_{1 i}, X_{1 i}^{2}, X_{2 i}, X_{2 i}^{2}, Y_{1 i}, Y_{1 i}^{2}\right)+\varepsilon_{2 i} \\
Y_{1 \mathrm{i}}=\beta_{10}+\beta_{11} X_{1 i}+\beta_{12} X_{1 i}^{2}+\delta_{11}\left(X_{1 i}-K_{11}\right)_{+}^{2}+\beta_{13} X_{2 i} \\
+\beta_{14} X_{2 i}^{2}+\delta_{12}\left(X_{2 i}-K_{21}\right)_{+}^{2}+\varepsilon_{1 i} \\
Y_{2 \mathrm{i}}=\beta_{20}+\beta_{21} X_{1 i}+\beta_{22} X_{1 i}^{2}+\delta_{21}\left(X_{1 i}-K_{11}\right)_{+}^{2}+\beta_{23} X_{2 i} \\
+\beta_{24} X_{2 i}^{2}+\delta_{22}\left(X_{2 i}-K_{21}\right)_{+}^{2}+\beta_{25} Y_{1 i}+\beta_{26} Y_{1 i}^{2} \\
+\delta_{23}\left(Y_{1 i}-K_{31}\right)_{+}^{2}+\varepsilon_{2 i}
\end{gathered}
$$

\section{Proof}

Before obtaining a model in quadratic nonparametric path analysis, the first model is obtained from (a) quadratic path analysis; and (b) linear nonparametric path analysis as follows:
First part:

It is known that the quadratic path analysis model with equation (11) and the model in (12)

$$
\begin{gathered}
Y_{1 i}=f_{1}\left(X_{1 i}, X_{1 i}^{2}, X_{2 i}, X_{2 i}^{2}\right)+\varepsilon_{1 i} \\
Y_{2 i}=f_{2}\left(X_{1 i}, X_{1 i}^{2}, X_{2 i}, X_{2 i}^{2}, Y_{1 i}, Y_{1 i}^{2}\right)+\varepsilon_{2 i} \\
Y_{1 \mathrm{i}}=\beta_{10}+\beta_{11} X_{1 i}+\beta_{12} X_{1 i}^{2}+\beta_{13} X_{2 i}+\beta_{14} X_{2 i}^{2}+\varepsilon_{1 i} \\
Y_{2 \mathrm{i}}=\beta_{20}+\beta_{21} X_{1 i}+\beta_{22} X_{1 i}^{2}+\beta_{23} X_{2 i}+\beta_{24} X_{2 i}^{2}+\beta_{25} Y_{1 i} \\
+\beta_{26} Y_{1 i}^{2}+\varepsilon_{2 i}
\end{gathered}
$$

The second part:

Obtained from the results of Lemma 3.1 which is a form of linear truncated spline nonparametric path analysis presented in equations (11) and (12) with the following matrix form:

$$
\begin{gathered}
\boldsymbol{Y}_{2 \mathrm{nx} 1}=\boldsymbol{X}_{2 n \times 12} \boldsymbol{\beta}_{12 x 1}+\varepsilon_{2 n x 1} \\
{\left[\begin{array}{c}
Y_{11} \\
Y_{12} \\
\vdots \\
Y_{1 n} \\
Y_{21} \\
Y_{22} \\
\vdots \\
Y_{2 n}
\end{array}\right]=\left[\begin{array}{cc}
\boldsymbol{X}_{\boldsymbol{X}} & \mathbf{0}_{n x 7} \\
\mathbf{0}_{n x 5} & \boldsymbol{X}_{\boldsymbol{X}}
\end{array}\right]\left[\begin{array}{c}
\beta_{10} \\
\beta_{11} \\
\delta_{11} \\
\beta_{12} \\
\delta_{12} \\
\beta_{20} \\
\beta_{21} \\
\delta_{21} \\
\beta_{22} \\
\delta_{22} \\
\beta_{23} \\
\delta_{23}
\end{array}\right]+\left[\begin{array}{c}
\varepsilon_{11} \\
\varepsilon_{11} \\
\vdots \\
\varepsilon_{1 n} \\
\varepsilon_{21} \\
\varepsilon_{22} \\
\vdots \\
\varepsilon_{2 n}
\end{array}\right] ;}
\end{gathered}
$$

Where,

$$
\begin{gathered}
\boldsymbol{X}_{\boldsymbol{X}}=\left[\begin{array}{ccccc}
1 & X_{11} & \left(X_{11}-K_{11}\right)_{+} & X_{21} & \left(X_{21}-K_{21}\right)_{+} \\
1 & X_{12} & \left(X_{12}-K_{11}\right)_{+} & X_{22} & \left(X_{22}-K_{21}\right)_{+} \\
\vdots & \vdots & \vdots & \vdots & \vdots \\
1 & X_{1 n} & \left(X_{1 n}-K_{11}\right)_{+} & X_{2 n} & \left(X_{2 n}-K_{21}\right)_{+}
\end{array}\right] \\
\boldsymbol{X}_{X Y}=\left[\begin{array}{ccccc}
1 & X_{11} & \left(X_{11}-K_{11}\right)_{+} & X_{21} & \left(X_{21}-K_{21}\right)_{+} \\
1 & X_{12} & \left(X_{12}-K_{11}\right)_{+} & X_{22} & \left(X_{22}-K_{21}\right)_{+} \\
\vdots & \vdots & \vdots & \vdots & \vdots \\
1 & X_{1 n} & \left(X_{1 n}-K_{11}\right)_{+} & X_{2 n} & \left(X_{2 n}-K_{21}\right)_{+} \\
& & Y_{11} & \left(Y_{11}-K_{31}\right)_{+} \\
& & Y_{12} & \left(Y_{12}-K_{31}\right)_{+} \\
& \vdots & \vdots \\
& & Y_{1 n} & \left(Y_{1 n}-K_{31}\right)_{+}
\end{array}\right]
\end{gathered}
$$

From the equation in the quadratic path analysis model, and the results in Lemma 3.1, a function is formed as in equations (9) and (10), so that the following matrix is obtained: 


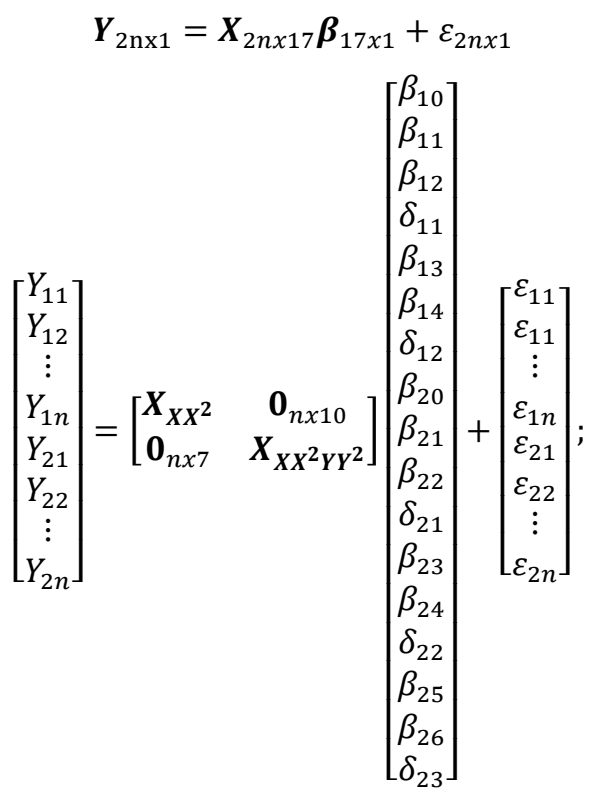

Where,

$$
\begin{aligned}
& =\left[\begin{array}{ccccccc}
1 & X_{11} & X_{11}^{2} & \left(X_{11}-K_{11}\right)_{+}^{2} & X_{21} & X_{21}^{2} & \left(X_{11}-K_{21}\right)_{+}^{2} \\
1 & X_{12} & X_{12}^{2} & \left(X_{12}-K_{11}\right)_{+}^{2} & X_{22} & X_{22}^{2} & \left(X_{12}-K_{21}\right)_{+}^{2} \\
\vdots & \vdots & \vdots & \vdots & \vdots & \vdots & \vdots \\
1 & X_{1 n} & X_{1 n}^{2} & \left(X_{1 n}-K_{11}\right)_{+}^{2} & X_{2 n} & X_{2 n}^{2} & \left(X_{1 n}-K_{2 n}\right)_{+}^{2}
\end{array}\right] \\
& =\left[\begin{array}{ccccccc}
1 & X_{11} & X_{11}^{2} & \left(X_{11}-K_{11}\right)_{+}^{2} & X_{21} & X_{21}^{2} & \left(X_{21}-K_{21}\right)_{+}^{2} \\
1 & X_{12} & X_{12}^{2} & \left(X_{12}-K_{11}\right)_{+}^{2} & X_{22} & X_{22}^{2} & \left(X_{22}-K_{21}\right)_{+}^{2} \\
\vdots & \vdots & \vdots & \vdots & \vdots & \vdots & \vdots \\
1 & X_{1 n} & X_{1 n}^{2} & \left(X_{1 n}-K_{11}\right)_{+}^{2} & X_{2 n} & X_{2 n}^{2} & \left(X_{2 n}-K_{2 n}\right)_{+}^{2}
\end{array}\right. \\
& Y_{11} \quad Y_{11}^{2} \quad\left(Y_{11}-K_{31}\right)_{+}^{2} \\
& Y_{12} \quad Y_{12}^{2} \quad\left(Y_{12}-K_{31}\right)_{+}^{2} \\
& \begin{array}{clc}
\vdots & \vdots & \vdots \\
Y_{1 n} & Y_{1 n}^{2} & \left(Y_{1 n}-K_{31}\right)_{+}^{2}
\end{array}
\end{aligned}
$$

Lemma 3.3 Forms of Cubic Truncated Spline Nonparametric Path Analysis Model

If given paired data $\left(X_{1 i}, X_{1 i}^{2}, X_{1 i}^{3}, X_{2 i}, X_{2 i}^{2}, X_{2 i}^{3}\right.$, $\left.Y_{1 i}, Y_{1 i}^{2}, Y_{1 i}^{3}, Y_{2 i}, Y_{2 i}^{2}, Y_{2 i}^{3}\right)$ with $i=1,2, \ldots, n$ those that follow the nonparametric path analysis model, then the form of the cubic nonparametric path analysis function is obtained as presented in equation (13) and the model in (14).

$$
\begin{gathered}
Y_{1 i}=f_{1}\left(X_{1 i}, X_{1 i}^{2}, X_{1 i}^{3}, X_{2 i}, X_{2 i}^{2}, X_{2 i}^{3}\right)+\varepsilon_{1 i} \\
Y_{2 i}=f_{2}\left(X_{1 i}, X_{1 i}^{2}, X_{1 i}^{3}, X_{2 i}, X_{2 i}^{2}, X_{2 i}^{3}, Y_{1 i}, Y_{1 i}^{2}, Y_{1 i}^{3}\right)+\varepsilon_{2 i}(13) \\
Y_{1 \mathrm{i}}=\beta_{10}+\beta_{11} X_{1 i}+\beta_{12} X_{1 i}^{2}+\beta_{13} X_{1 i}^{2}+\delta_{11}\left(X_{1 i}-K_{11}\right)_{+}^{3} \\
+\beta_{14} X_{2 i}+\beta_{15} X_{2 i}^{2}+\beta_{16} X_{2 i}^{2}+\delta_{12}\left(X_{2 i}-K_{21}\right)_{+}^{3}+\varepsilon_{1 i} \\
Y_{2 \mathrm{i}}=\beta_{20}+\beta_{21} X_{1 i}+\beta_{22} X_{1 i}^{2}+\beta_{23} X_{1 i}^{3}+\delta_{21}\left(X_{1 i}-K_{11}\right)_{+}^{3} \\
+\beta_{24} X_{2 i}+\beta_{25} X_{2 i}^{2}+\beta_{26} X_{2 i}^{3}+\delta_{22}\left(X_{2 i}-K_{21}\right)_{+}^{2} \\
+\beta_{27} Y_{1 i}+\beta_{28} Y_{1 i}^{2}+\beta_{29} Y_{1 i}^{3}+\delta_{23}\left(Y_{1 i}-K_{31}\right)_{+}^{3}+\varepsilon_{2 i}
\end{gathered}
$$

\section{Proof}

From the results in Lemma 3.1 and Lemma 3.2, an expansion can be made by adding the cubic order, so we can obtain a function that is formed as in equations (13) and (14), the following matrix is obtained:

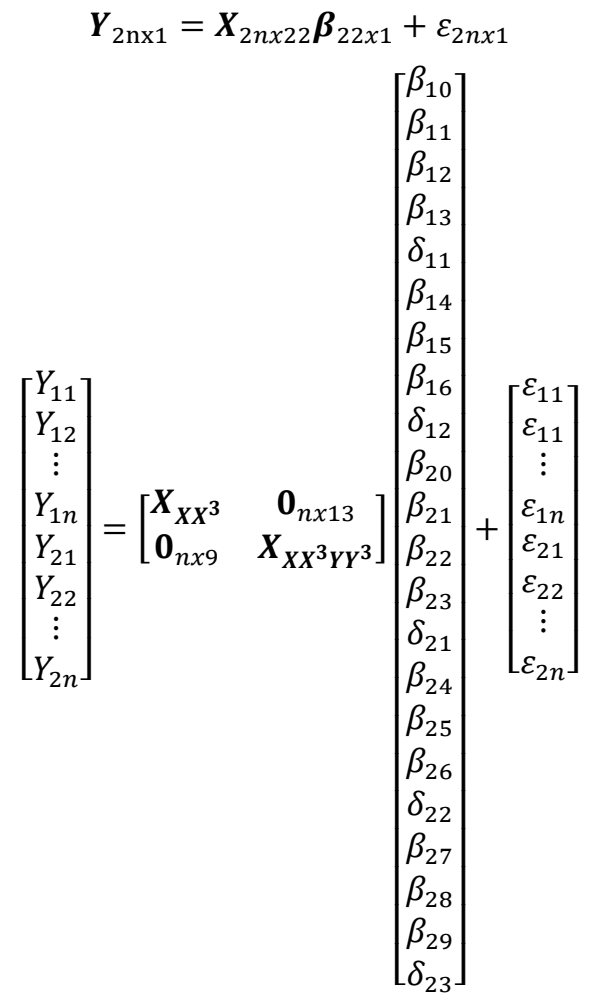

Where,

$$
\begin{aligned}
& X_{X X^{3}}= \\
& {\left[\begin{array}{ccccccccc}
1 & X_{11} & X_{11}^{2} & X_{11}^{3} & \left(X_{11}-K_{11}\right)_{+}^{3} & X_{21} & X_{21}^{2} & X_{21}^{3} & \left(X_{21}-K_{21}\right)_{+}^{3}
\end{array}\right]} \\
& \begin{array}{lllllllll}
1 & X_{12} & X_{12}^{2} & X_{12}^{2} & \left(X_{12}-K_{11}\right)_{+}^{3} & X_{22} & X_{22}^{2} & X_{22}^{3} & \left(X_{22}-K_{21}\right)_{+}^{3}
\end{array} \\
& \begin{array}{ccccccccc}
\vdots & \vdots & \vdots & \vdots & \vdots & \vdots & \vdots & \vdots & \vdots \\
1 & X_{1 n} & X_{1 n}^{2} & X_{1 n}^{2} & \left(X_{1 n}-K_{11}\right)_{+}^{3} & X_{2 n} & X_{2 n}^{2} & X_{2 n}^{3} & \left(X_{2 n}-K_{2 n}\right)_{+}^{3}
\end{array} \\
& \boldsymbol{X}_{X X^{3} Y Y^{3}}= \\
& {\left[\begin{array}{ccccccccc}
1 & X_{11} & X_{11}^{2} & X_{11}^{3} & \left(X_{11}-K_{11}\right)_{+}^{3} & X_{21} & X_{21}^{2} & X_{21}^{3} & \left(X_{21}-K_{21}\right)_{+}^{3}
\end{array}\right.} \\
& \begin{array}{ccccccccc}
1 & X_{12} & X_{12}^{2} & X_{12}^{2} & \left(X_{12}-K_{11}\right)_{+}^{3} & X_{22} & X_{22}^{2} & X_{22}^{3} & \left(X_{22}-K_{21}\right)_{+}^{3} \\
\vdots & \vdots & \vdots & \vdots & \vdots & \vdots & \vdots & \vdots & \vdots
\end{array} \\
& \begin{array}{ccccccccc}
\vdots & \vdots & \vdots & \vdots & \vdots & \vdots & \vdots & \vdots & \vdots \\
1 & X_{1 n} & X_{1 n}^{2} & X_{1 n}^{2} & \left(X_{1 n}-K_{11}\right)_{+}^{3} & X_{2 n} & X_{2 n}^{2} & X_{2 n}^{3} & \left(X_{2 n}-K_{2 n}\right)_{+}^{3}
\end{array} \\
& \left.\begin{array}{cccc}
Y_{11} & Y_{11}^{2} & Y_{11}^{3} & \left(Y_{11}-K_{31}\right)_{+}^{2} \\
Y_{12} & Y_{12}^{2} & Y_{12}^{3} & \left(Y_{12}-K_{31}\right)_{+}^{2} \\
\vdots & \vdots & \vdots & \vdots \\
Y_{1 n} & Y_{1 n}^{2} & Y_{1 n}^{3} & \left(Y_{1 n}-K_{31}\right)_{+}^{2}
\end{array}\right]
\end{aligned}
$$

Theorem

If paired data are given following the nonparametric path model in the cross-section data that fulfill the form of the nonparametric path function for data cross section as presented in Lemma 3.1, Lemma 3.2, and Lemma 3.3, the truncated spline estimator method that minimizes the number of squares of error using the Ordinary Least Square method is presented. as follows: 


$$
\operatorname{Min}_{\boldsymbol{\beta} \in R^{k+r+1}}\left\{\boldsymbol{\varepsilon}^{t} \boldsymbol{\varepsilon}\right\}=\operatorname{Min}_{\boldsymbol{\beta} \in R^{k+r+1}}\left\{\left(\begin{array}{c}
\varepsilon_{1} \\
\varepsilon_{2} \\
\vdots \\
\varepsilon_{n}
\end{array}\right)^{t}\left(\begin{array}{c}
\varepsilon_{1} \\
\varepsilon_{2} \\
\vdots \\
\varepsilon_{n}
\end{array}\right)\right\}
$$

lowered against $\underset{\hat{\beta}}{\hat{\alpha}}$ and then equated with zero, then:

$$
\begin{gathered}
\frac{\partial \boldsymbol{\varepsilon}^{t} \boldsymbol{\varepsilon}}{\partial \boldsymbol{\beta}}=\frac{\boldsymbol{Y}^{t} \boldsymbol{Y}-2 \widehat{\boldsymbol{\beta}}^{t} \boldsymbol{X}[\mathbf{K}]^{t} \boldsymbol{Y}+\widehat{\boldsymbol{\beta}}^{t} \boldsymbol{X}[\mathbf{K}]^{t} \boldsymbol{X}[\mathbf{K}] \widehat{\boldsymbol{\beta}}}{\partial \boldsymbol{\beta}}=0 \\
\widehat{\boldsymbol{\beta}}=\left(\boldsymbol{X}[\mathbf{K}]^{t} \boldsymbol{X}[\mathbf{K}]\right)^{-\mathbf{1}} \boldsymbol{X}[\mathbf{K}]^{t} \boldsymbol{Y}
\end{gathered}
$$

\section{Proof}

In the form of a matrix, the nonparametric path is expressed as follows:

$$
\left[\begin{array}{c}
Y_{1} \\
Y_{2} \\
\vdots \\
Y_{n}
\end{array}\right]_{n x 1}=\left[\begin{array}{c}
f\left(X_{1}\right) \\
f\left(X_{2}\right) \\
\vdots \\
f\left(X_{n}\right)
\end{array}\right]_{n x 1}+\left[\begin{array}{c}
\mathcal{E}_{1} \\
\mathcal{E}_{2} \\
\vdots \\
\mathcal{E}_{n}
\end{array}\right]_{n x 1}
$$

Truncated spline nonparametric regression curve equation for $i=1,2, \ldots, n$ written in the form of an equation as follows:

$$
\begin{aligned}
& {\left[\begin{array}{c}
f\left(X_{1}\right) \\
f\left(X_{2}\right) \\
\vdots \\
f\left(X_{n}\right)
\end{array}\right]} \\
& =\left[\begin{array}{ccccccc}
1 & \mathrm{X}_{1} & \cdots & \mathrm{X}_{1}^{q} & \left(\mathrm{X}_{1}-\mathrm{K}_{1}\right)_{+}^{q} & \cdots & \left(\mathrm{X}_{1}-\mathrm{K}_{r}\right)_{+}^{q} \\
1 & \mathrm{X}_{2} & \cdots & \mathrm{X}_{2}^{q} & \left(\mathrm{X}_{2}-\mathrm{K}_{1}\right)_{+}^{q} & \cdots & \left(\mathrm{X}_{2}-\mathrm{K}_{r}\right)_{+}^{q} \\
\vdots & \vdots & \ddots & \vdots & \vdots & \ddots & \vdots \\
1 & \mathrm{X}_{n} & \cdots & \mathrm{X}_{n}^{q} & \left(\mathrm{X}_{n}-\mathrm{K}_{1}\right)_{+}^{q} & \cdots & \left(\mathrm{X}_{n}-\mathrm{K}_{r}\right)_{+}^{q}
\end{array}\right]
\end{aligned}
$$

$$
\left[\begin{array}{c}
\beta_{0} \\
\beta_{1} \\
\beta_{2} \\
\vdots \\
\beta_{k} \\
\delta_{1} \\
\vdots \\
\delta_{r}
\end{array}\right]
$$

It can also be written as:

$$
\underset{\sim}{f}(X)=\mathbf{X}(\underset{\sim}{K}) \underset{\sim}{\beta}
$$

Based on the nonparametric regression model, the truncated spline function of order $\mathrm{m}$ with knots points $\mathrm{K}_{1}$, $\mathrm{K}_{2}, \ldots, \mathrm{K}_{\mathrm{h}}$. assumed to be normally distributed, independent of each other with a mean value of zero $\varepsilon_{i}$ and a variance of $\sigma^{2}$, so that $\varepsilon_{i} \sim N\left(0, \sigma^{2}\right)$. Nonparametric regression curve estimation can be done by using the Ordinary Least Square (OLS) method. In its estimation, OLS minimizes the number of squares of errors, where the error is obtained from equation (15).

$$
\varepsilon_{i}=Y_{i}-f\left(X_{i}\right)
$$

Equation (19) can be expressed in matrix notation as follows:

$$
\underset{\sim}{\varepsilon}=\underset{\sim}{Y}-\underset{\sim}{f}(X)
$$

The parameter estimates obtained by the OLS method are as follows:

$$
\begin{aligned}
& \dot{\varepsilon}_{\sim}^{\mathrm{T}} \mathcal{\varepsilon}=(\underset{\sim}{Y}-\underset{\sim}{f}(X))^{\mathrm{T}}(\underset{\sim}{Y}-\underset{\sim}{f}(X)) \\
& =(\underset{\sim}{Y}-\mathbf{X}(\underset{\sim}{K}) \underset{\sim}{\beta})^{\mathrm{T}}(\underset{\sim}{Y}-\mathbf{X}(\underset{\sim}{K}) \underset{\sim}{\beta}) \\
& =\left({\underset{\sim}{T}}^{\mathrm{T}}-\mathbf{X}(\underset{\sim}{K})^{\mathrm{T}} \underset{\sim}{\beta^{\mathrm{T}}}\right)(\underset{\sim}{Y}-\mathbf{X}(\underset{\sim}{K}) \underset{\sim}{\beta}) \\
& =Y_{\sim}^{\mathrm{T}} Y \sim{ }_{\sim}-\underline{\sim}^{\mathrm{T}} \mathbf{X}(\underset{\sim}{K}) \underset{\sim}{\beta}-{\underset{\sim}{\beta}}^{\mathrm{T}} \mathbf{X}(K)^{\mathrm{T}} \underset{\sim}{Y}+\mathbf{X}(K)^{\mathrm{T}}{ }_{\sim}^{\mathrm{T}}{ }_{\sim}^{\mathrm{T}} \mathbf{X}(\underset{\sim}{K}) \underset{\sim}{\beta} \\
& ={\underset{\sim}{Y}}_{\sim}^{\mathrm{T}} \underset{\sim}{Y}-2 \underset{\sim}{\beta^{\mathrm{T}}} \mathbf{X}(\underset{\sim}{K})^{\mathrm{T}} \underset{\sim}{\boldsymbol{\beta}}+\mathbf{X}(\underset{\sim}{K})^{\mathrm{T}} \underset{\sim}{\beta^{\mathrm{T}}} \mathbf{X}(\underset{\sim}{K}) \underset{\sim}{\beta}
\end{aligned}
$$

The parameter estimate is obtained by forming the normal equation from equation (21) as follows:

$$
\begin{aligned}
& \frac{\partial\left({\underset{\varepsilon}{\mathcal{E}} \mathcal{\varepsilon})}^{\mathrm{T}}\right)}{\partial(\hat{\sim})}=0
\end{aligned}
$$

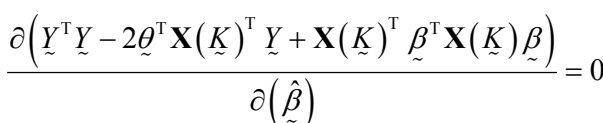

$$
\begin{aligned}
& -2 \mathbf{X}(\underset{\sim}{K})^{\mathrm{T}} \underset{\sim}{Y}+2 \mathbf{X}(\underset{\sim}{K})^{\mathrm{T}} \mathbf{X}(\underset{\sim}{K}) \underset{\sim}{\hat{\beta}}=0 \\
& -2\left(\mathbf{X}(\underset{\sim}{K})^{\mathrm{T}} \underset{\sim}{Y}-\mathbf{X}(\underset{\sim}{K})^{\mathrm{T}} \mathbf{X}(\underset{\sim}{K}) \hat{\beta}\right)=0 \\
& \mathbf{X}(K)^{\mathrm{T}} \underset{\sim}{Y}-\mathbf{X}(K)^{\mathrm{T}} \mathbf{X}(\underset{\sim}{K}) \hat{\beta}=0 \\
& \mathbf{X}(\underset{\sim}{K})^{\mathrm{T}} \mathbf{X}(\underset{\sim}{K}) \underset{\sim}{\hat{\beta}}=\mathbf{X}(\underset{\sim}{K})^{\mathrm{T}} \underset{\sim}{Y} \\
& \hat{\beta}=\left[\mathbf{X}(\underset{\sim}{K})^{\mathrm{T}} \mathbf{X}(\underset{\sim}{K})\right]^{-1}\left[\mathbf{X}(\underset{\sim}{K})^{\mathrm{T}} \underset{\sim}{Y}\right]
\end{aligned}
$$

Based on equation (15), the nonparametric path estimation is obtained based on the matrix notation in equation (18) which is as follows:

$$
\begin{gathered}
\hat{\sim} \underset{\sim}{f}(X)=\mathbf{X}(\underset{\sim}{K}) \underset{\sim}{\hat{\beta}} \\
\hat{\sim} \underset{\sim}{\hat{f}}(X)=\mathbf{X}(\underset{\sim}{K})\left[\mathbf{X}(\underset{\sim}{K})^{\mathrm{T}} \mathbf{X}(\underset{\sim}{K})\right]^{-1}\left[\mathbf{X}(\underset{\sim}{K})^{\mathrm{T}} \underset{\sim}{Y}\right]
\end{gathered}
$$

So equation (23) shows the curve estimate for the nonparametric path model with the truncated spline approach.

\subsection{Linearity Testing}

In statistical modeling, information is needed about the pattern of relationships between variables to determine whether a method is used through a parametric or nonparametric, or semiparametric approach. The results of linearity testing with the Ramsey RESET are as follows: 
Table 1. Ramsey RESET Linearity Test Results

\begin{tabular}{|l|l|l|}
\hline Variable & P-value & Relationship \\
\hline X1 to Y1 & $<0.0001$ & Nonlinear \\
\hline X2 to Y1 & 0.0263 & Nonlinear \\
\hline X1 to Y2 & 0.0009 & Nonlinear \\
\hline X2 to Y2 & 0.0061 & Nonlinear \\
\hline Y1 to Y2 & 0.0158 & Nonlinear \\
\hline
\end{tabular}

Based on Table 1, the test results show that $\mathrm{p}$-value $<\alpha$ (0.05), then it can be decided to reject $\mathrm{H}_{0}$. Therefore, the relationship between variables indicates that the data do not meet the linearity assumption.

\subsection{Truncated Spline Nonparametric Path Model Degree of Linear Polynomial (Order $p=1)$}

The truncated spline nonparametric path model at the moment is linear (order $p=1$ ) with 1 knot for two exogenous variables and two endogenous variables as follows:

$$
\begin{aligned}
\hat{f_{1 i}=} & 96.7248+0.1263 X_{1 i}-0.0729\left(X_{1 i}-65.76\right)_{+} \\
& -0.2559 X_{2 i}+0.3827\left(X_{2 i}-35.85\right)_{+} \\
\hat{f}_{2 i}= & 60.8009+0.1388 X_{1 i}-0.2725\left(X_{1 i}-65.76\right)_{+} \\
& +0.2390 X_{2 i}-0.4989\left(X_{2 i}-35.85\right)_{+}-0.6755 f_{1 i} \\
& +0.2728\left(f_{1 i}-95.45\right)_{+}
\end{aligned}
$$

The truncated spline nonparametric path model at the moment is linear (order $p=1$ ) with 2 knots for two exogenous variables and two endogenous variables as follows:

$$
\begin{aligned}
\hat{f}_{1 i}= & 96.8497+0.2116 X_{1 i}-0.2917\left(X_{1 i}-60.90\right)_{+} \\
& +0.1723\left(X_{1 i}-68.22\right)_{+}-0.4131 X_{2 i}+1.0052 \\
& \left(X_{2 i}-35.85\right)_{+}-0.6783\left(X_{2 i}-40.47\right)_{+} \\
\hat{f}_{2 i}= & -0.2406+0.1286 X_{1 i}-0.1038\left(X_{1 i}-60.90\right)_{+} \\
& -0.1813\left(X_{1 i}-68.22\right)_{+}+0.5313 X_{2 i}-1.1000\left(X_{2 i}-35.85\right)_{+} \\
& +0.3198\left(X_{2 i}-40.47\right)_{+}-0.1168 f_{1 i}-0.3452\left(f_{1 i}-95.45\right)_{+} \\
& -4.9569\left(f_{1 i}-99.72\right)_{+}
\end{aligned}
$$

\subsection{Truncated Spline Nonparametric Path Model Degree of Linear Polynomial (Order $p=2$ )}

The truncated spline nonparametric path model at the quadratic time (order $\mathrm{p}=2$ ) with 1 knot for two exogenous variables and two endogenous variables as follows:

$$
\begin{aligned}
\hat{f}_{1 i}= & 0.0842+2.4051 X_{1 i}-0.0205 X_{1 i}^{2}+0.0248\left(X_{1 i}-60.90\right)_{+}^{2} \\
& +1.4117 X_{2 i}-0.0189 X_{2 i}^{2}+0.0172\left(X_{2 i}-35.85\right)_{+}^{2} \\
\hat{f}_{2 i}= & 0.0130+0.3659 X_{1 i}-0.0024 X_{1 i}^{2}-0.0026\left(X_{1 i}-60.90\right)_{+}^{2} \\
& +0.2280 X_{2 i}-0.0036 X_{2 i}^{2}-0.0068\left(X_{2 i}-35.85\right)_{+}^{2}+0.5983 f_{1 i} \\
& -0.0068 f_{1 i}^{2}+0.0547\left(f_{1 i}-95.45\right)_{+}^{2}
\end{aligned}
$$

The truncated spline nonparametric path model at quadratic time (order $\mathrm{p}=2$ ) with 2 knots for two exogenous variables and two endogenous variables as follows:

$$
\begin{aligned}
\hat{f}_{1 i}= & 0.0932+2.5214 X_{1 i}-0.0221 X_{1 i}^{2}+0.0397\left(X_{1 i}-60.90\right)_{+}^{2} \\
& -0.0175\left(X_{1 i}-68.22\right)_{+}^{2}+1.4981 X_{2 i}-0.0228 X_{2 i}^{2}+0.0674 \\
& \left(X_{2 i}-35.85\right)_{+}^{2}-0.0609\left(X_{2 i}-40.47\right)_{+}^{2} \\
\hat{f}_{2 i}= & 0.0122+0.3313 X_{1 i}-0.0018 X_{1 i}^{2}-0.0092\left(X_{1 i}-60.90\right)_{+}^{2} \\
& -0.0082\left(X_{1 i}-68.22\right)_{+}^{2}+0.2021 X_{2 i}-0.0018 X_{2 i}^{2}-0.0337 \\
& \left(X_{2 i}-35.85\right)_{+}^{2}+0.0332\left(X_{2 i}-40.47\right)_{+}^{2}+0.5631 f_{1 i} \\
& -0.0065 f_{1 i}^{2}+0.0486\left(f_{1 i}-95.45\right)_{+}^{2}
\end{aligned}
$$

\subsection{Truncated Spline Nonparametric Path Model Degree of Linear Polynomial (Order $p=3)$}

The truncated spline nonparametric path model at cubic time (order $\mathrm{p}=3$ ) with 1 knot for two exogenous variables and two endogenous variables as follows:

$$
\begin{aligned}
\hat{f_{1 i}=} & 0.0001+0.0019 X_{1 i}+0.0621 X_{1 i}^{2}-0.0006 X_{1 i}^{3} \\
& +0.0020\left(X_{1 i}-60.90\right)_{+}^{3}+0.0017 X_{2 i}+0.0225 X_{2 i}^{2} \\
& -0.0003 X_{2 i}^{3}-0.0002\left(X_{2 i}-43.51\right)_{+}^{3} \\
\hat{f}_{2 i}= & 0.0001+0.0001 X_{1 i}+0.0036 X_{1 i}^{2}-0.0001 X_{1 i}^{3} \\
& -0.0001\left(X_{1 i}-60.90\right)_{+}^{3}+0.0001 X_{2 i}+0.0015 X_{2 i}^{2} \\
& -0.0001 X_{2 i}^{3}+0.0001\left(X_{2 i}-43.51\right)_{+}^{3}+0.0002 f_{1 i} \\
& -0.0088 f_{1 i}^{2}-0.0001 f_{1 i}^{3}+0.0000\left(f_{1 i}-98.65\right)_{+}^{3}
\end{aligned}
$$

The truncated spline nonparametric path model at cubic time (order $p=3$ ) with 2 knots for two exogenous variables and two endogenous variables as follows:

$$
\begin{aligned}
\hat{f}_{1 i}= & 0.0001+0.0025 X_{1 i}+0.0711 X_{1 i}^{2}-0.0008 X_{1 i}^{3} \\
& +0.0071\left(X_{1 i}-60.90\right)_{+}^{3}-0.0078\left(X_{1 i}-68.22\right)_{+}^{3} \\
& +0.0015 X_{2 i}+0.0274 X_{2 i}^{2}-0.0004 X_{2 i}^{3}-0.0041 \\
& \left(X_{2 i}-36.67\right)_{+}^{3}+0.0762\left(X_{2 i}-40.58\right)_{+}^{3} \\
\hat{f}_{2 i}= & 0.0001+0.0001 X_{1 i}+0.0029 X_{1 i}^{2}-0.0001 X_{1 i}^{3} \\
& -0.0001\left(X_{1 i}-60.90\right)_{+}^{3}+0.0001\left(X_{1 i}-68.22\right)_{+}^{3} \\
& +0.0001 X_{2 i}+0.0014 X_{2 i}^{2}-0.0001 X_{2 i}^{3}-0.0004 \\
& \left(X_{2 i}-36.67\right)_{+}^{3}+0.0005\left(X_{2 i}-40.58\right)_{+}^{3}+0.0002 f_{1 i} \\
& -0.0087 f_{1 i}^{2}-0.0001 f_{1 i}^{3}-0.0006\left(f_{1 i}-95.57\right)_{+}^{3} \\
& -0.0001\left(f_{1 i}-97.88\right)_{+}^{3}
\end{aligned}
$$

\subsection{Best Order Polynomial Truncated Spline Nonparametric Model}

The best truncated spline nonparametric path model is obtained if the knot point is optimal. Meanwhile, to obtain the optimal knot point, you must find the smallest GCV value. The estimation of the nonparametric truncated spline path model for the linear, quadratic, and cubic orders with 1 knot point and 2 best knot point is as follows: 
Table 2. Estimation of Best Truncated Spline Nonparametric Path Functions

\begin{tabular}{|c|c|c|c|}
\hline Order & Knots & $\mathbf{R}^{2}$ & GCV \\
\hline Linear & 1 & 0.9621 & 25.9720 \\
\hline Linear & 2 & 0.9696 .9059 \\
\hline Quadratic & 1 & 0.9609 & 27.1042 \\
\hline Quadratic & 2 & 0.9613 & 26.8356 \\
\hline Cubic & 1 & 0.9587 & 38.1283 \\
\hline Cubic & 2 & 0.9595 & 32.5142 \\
\hline
\end{tabular}

Based on Table 2, it is known that the smallest GCV value of 25,9059 and the largest $R^{2}$ value of 0.9696 is obtained in the nonparametric path model truncated spline degree of linear polynomial (order $\mathrm{p}=2$ ) with 2 knots. The model has an $\mathrm{R}^{2}$ value of $96.96 \%$. That is, the model that has been formed explains the diversity of endogenous variables by $96.96 \%$, and the rest is explained by other factors that cannot be known in the model by $3.04 \%$. The effect of the best model estimation results is obtained as follows:

$$
\begin{aligned}
\hat{f}_{1 i}= & 96.8497+0.2116 X_{1 i}-0.2917\left(X_{1 i}-60.90\right)_{+} \\
& +0.1723\left(X_{1 i}-68.22\right)_{+}-0.4131 X_{2 i}+1.0052 \\
& \left(X_{2 i}-35.85\right)_{+}-0.6783\left(X_{2 i}-40.47\right)_{+} \\
\hat{f}_{2 i}= & -0.2406+0.1286 X_{1 i}-0.1038\left(X_{1 i}-60.90\right)_{+} \\
& -0.1813\left(X_{1 i}-68.22\right)_{+}+0.5313 X_{2 i}-1.1000 \\
& \left(X_{2 i}-35.85\right)_{+}+0.3198\left(X_{2 i}-40.47\right)_{+}-0.1168 f_{1 i} \\
& -0.3452\left(f_{1 i}-95.45\right)_{+}-4.9569\left(f_{1 i}-99.72\right)_{+}
\end{aligned}
$$

The nonparametric path model truncated spline degree of linear polynomial (order $p=1$ ) with 2 point knots has several relationships. The relationship between the variable quality service $\left(\mathrm{X}_{1}\right)$ and the variable willingness to pay $\left(\mathrm{Y}_{1}\right)$, the relationship between the variable lifestyle $\left(\mathrm{X}_{2}\right)$ and the variable willingness to pay $\left(\mathrm{Y}_{1}\right)$, the relationship between the variable quality service $\left(\mathrm{X}_{1}\right)$ with the variable on time to pay $\left(\mathrm{Y}_{2}\right)$, the relationship between the variable lifestyle $\left(\mathrm{X}_{2}\right)$ and the variable on time to pay $\left(\mathrm{Y}_{2}\right)$, and the relationship between the variable willingness to pay $\left(\mathrm{Y}_{1}\right)$ and the variable on time to pay $\left(\mathrm{Y}_{2}\right)$. In the form of an image, only the relationship between one exogenous variable and one endogenous variable is presented to facilitate discussion. In the form of an image, the nonparametric path model is cut by a degree of linear polynomial spline (order $\mathrm{p}=1$ ) with 2-point knots presented as follows: 


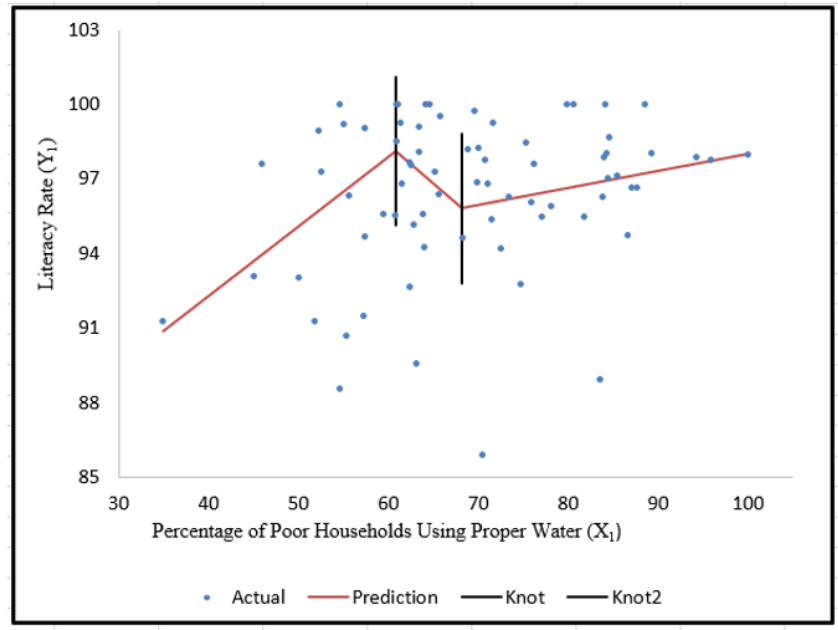

The Relationship Between $\mathrm{X}_{1}$ to $\mathrm{Y}_{1}$

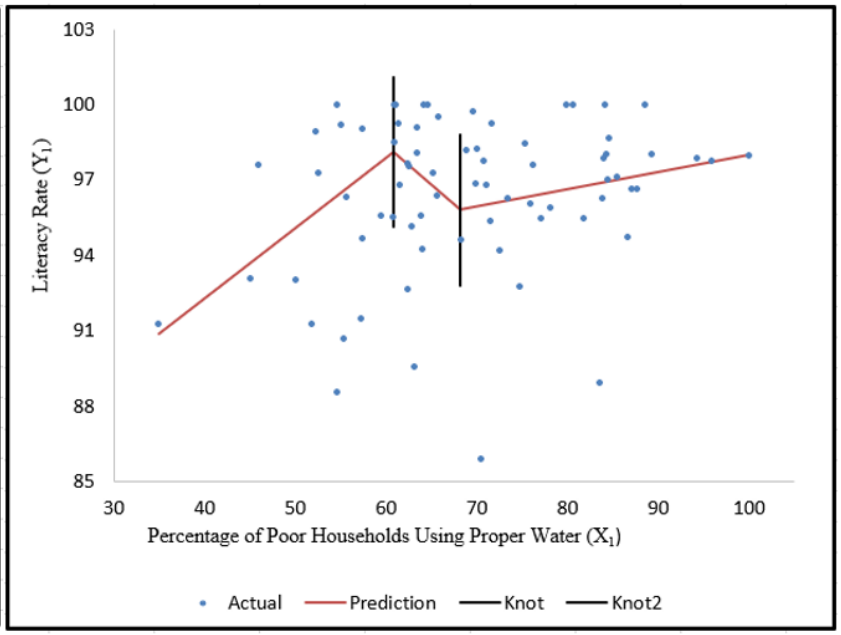

The Relationship Between $\mathrm{X}_{2}$ to $\mathrm{Y}_{1}$

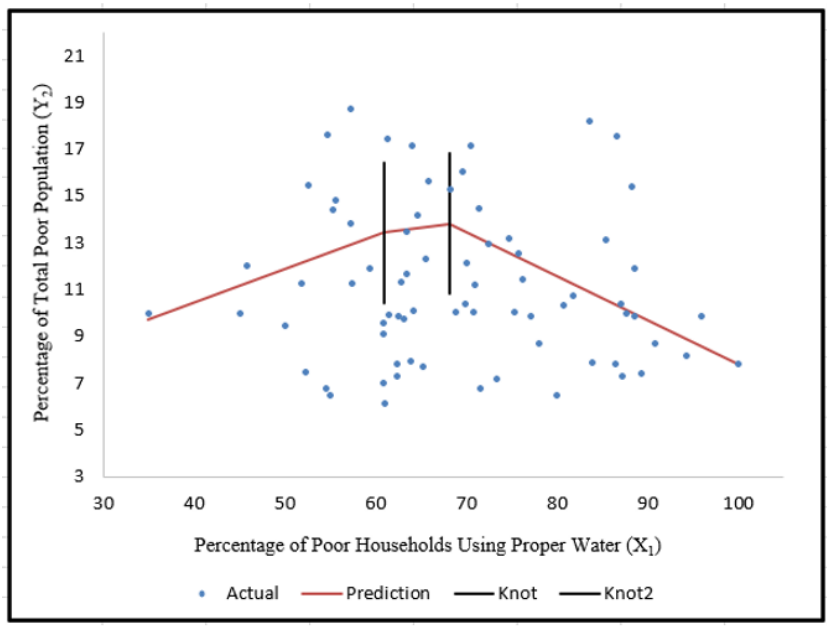

The Relationship Between $\mathrm{X}_{1}$ to $\mathrm{Y}_{2}$

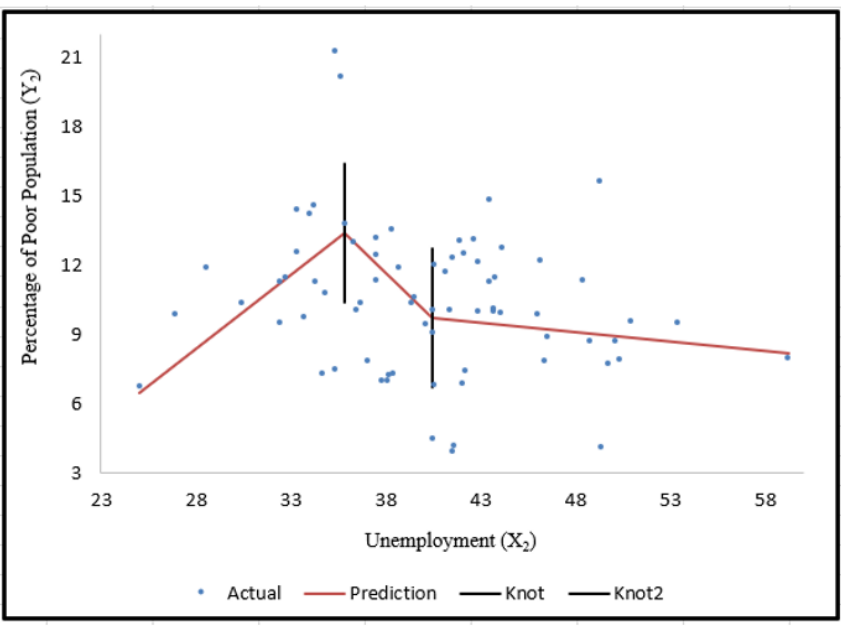

The Relationship Between $\mathrm{X}_{2}$ to $\mathrm{Y}_{2}$

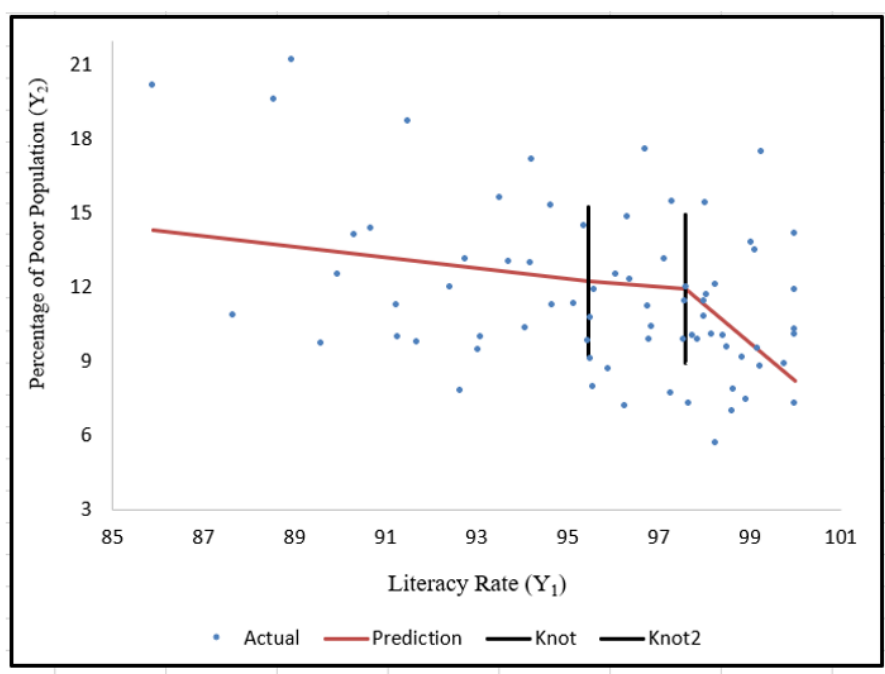

The Relationship Between $\mathrm{X}_{2}$ to $\mathrm{Y}_{2}$

Figure 2. Functions of Truncated Spline Linear Nonparametric Path Model 2 Knots

The truncated spline linear 2 points knot nonparametric path model is as follows: 


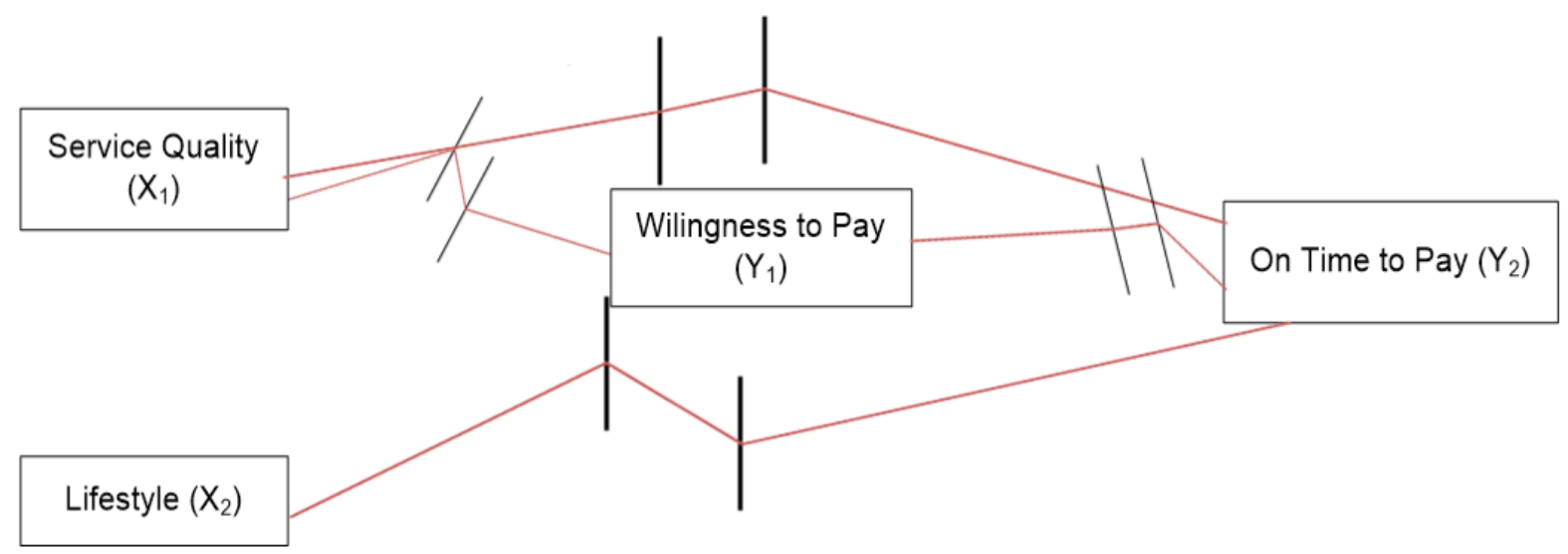

Figure 3. Truncated Spline Linear 2 Knot Nonparametric Path Model

\subsection{Hypothesis Test}

The linear hypothesis testing of parameters in the best model of path truncated spline analysis is used to determine whether there is an effect of exogenous variables on endogenous variables. The test was carried out on the best model, namely the linear order truncated spline path model with 2 knots point. Analysis results of hypothesis testing for the truncated spline path model, the degree of two knots linear polynomials is presented in Table 3.

Table 3. Results of Testing the Linear Parameter Hypothesis

\begin{tabular}{|c|c|c|c|}
\hline Function & P-value & Decision & Conclusion \\
\hline $\mathrm{X} 1$ to $\mathrm{Y} 1$ & $<0.0001$ & Reject $\mathrm{H}_{0}$ & Significant \\
\hline $\mathrm{X} 1$ to $\mathrm{Y} 2$ & 0.0003 & Reject $\mathrm{H}_{0}$ & Significant \\
\hline $\mathrm{X} 2$ to $\mathrm{Y} 1$ & $<0.0001$ & Reject $\mathrm{H}_{0}$ & Significant \\
\hline $\mathrm{X} 2$ to $\mathrm{Y} 2$ & 0.0051 & Reject $\mathrm{H}_{0}$ & Significant \\
\hline $\mathrm{Y} 1$ to $\mathrm{Y} 2$ & 0.9999 & Accept $\mathrm{H}_{0}$ & Not significant \\
\hline
\end{tabular}

Based on Table 3, the results of hypothesis testing on function estimates that have a significant effect on the relationship between service quality and willingness to pay, the relationship between service quality and on time to pay, the relationship between lifestyle and willingness to pay, and the relationship between lifestyle and on time pay. While testing the hypothesis on the estimation of the relationship function which is not significant when the relationship between willingness to pay and on time to pay.

\section{Conclusions}

Based on the results of the analysis and discussion that has been done, it can be concluded that:

1. Estimation of nonparametric truncated spline function of linear, quadratic, and cubic 1 and 2 knot points on the variables of service quality, lifestyle, and willingness to pay that affect the variable on time to pay. The best model is obtained on a nonparametric truncated spline linear path model with 2 knot points. This model has the smallest GCV value compared to other models, which is 25.9059 and the $\mathrm{R}^{2}$ value is $96.96 \%$.

2. The results of hypothesis testing indicate that there are four relationships that have a significant effect, namely the relationship between service quality and willingness to pay, the relationship between service quality and punctuality in paying, the relationship between lifestyle and willingness to pay, and the relationship between lifestyle and punctuality in paying. Meanwhile, the estimated function that has no significant effect on the relationship between willingness to pay and on time to pay.

\section{REFERENCES}

[1] Uyanik GK, Güler N. "A Study on Multiple Linear Regression Analysis," Procedia - Social and Behavioral Sciences, vol. 106, pp. 234-240, 2013. DOI: 10.1016/j.sbspro.2013.12.027

[2] Widyastuti, D. A., Fernandes, A. A. R., Pramoedyo, H., Nurjannah, and Solimun. "Test Efficiency Analysis of Parametric, Nonparametric, Semiparametric Regression in Spatial Data," Mathematics and Statistics, vol. 8, no. 5, pp. 506-519, 2020. DOI: $10.13189 / \mathrm{ms} .2020 .080503$

[3] Hidayat, R, Yuliani Y, and Marwan S. "Model Regresi Nonparametrik dengan Pendekatan Spline Truncated." Prosiding 3, no. 1, 2018.

[4] Suparti, S., and Prahutama A. "Pemodelan Regresi Nonparametrik Menggunakan Pendekatan Polinomial Lokal pada Beban Listrik di Kota Semarang," Media Statistika, vol. 9, no. 2, pp. 85-93, 2016.

[5] Fernandes, A. A. R., Widyastuti, D. A., Nurjannah. "Smoothing Spline Semiparametric Regression Model Assumption Using PWLS Approach,” International Journal of Advanced Science and Technology, vol. 29, no. 4, pp. 2059-2070, 2020. 
[6] S. Sudaryono. "Application Analysis (Path Analysis) based on the order of placement of variables in the study," Journal of Education and Culture, vol. 17, no. 4, pp. 391-403, 2011. DOI: $10.24832 /$ jpnk.v17i4.36

[7] Fariq H. M., and Fernandes, A. A. R. "Estimation of Truncated Spline Function in Non-parametric Path Analysis Based on Weighted Least Square (WLS)," In Materials Science and Engineering Conference Series, vol. 546, no. 5, p. 052027,2019 , DOI: $10.1088 / 1757-899 X / 546 / 5 / 052027$.
[8] Eubank RL, C. Huang, Maldonado YM, N. Wang, S. Wang, Buchanan RJ. "Smoothing Spline Estimation in Varying-Coefficient Models," JR Statist. Soc., vol. 66, no. 3, pp. 653-667, 2004. DOI: doi:10.1111/j.1467-9868.2004.b5 595.x

[9] Husnawaty, Husnawaty. "Kualitas Pelayanan Pemberian Kredit terhadap Peningkatan Kredit Pemilikan Rumah (KPR)." Bongaya Journal for Research in Accounting (BJRA) 3, no. 1, pp. 65-75, 2020. 